\title{
Mycobacterium chimaera Disseminated Infection
}

\author{
Ruben de Melo Carvalho ${ }^{\mathrm{a}, \mathrm{c}}$, Ana Luisa Nunes ${ }^{\mathrm{b}}$, Rosa Sa ${ }^{\mathrm{a}}$, Isabel Ramos ${ }^{\mathrm{a}}$, \\ Cristina Valente ${ }^{\mathrm{a}}$, Jose Saraiva da Cunha ${ }^{\mathrm{a}}$
}

\begin{abstract}
The incidence and prevalence of nontuberculous mycobacterial disease is increasing due to enhanced clinician awareness and improved detection methods. The species identification using molecular microbiology techniques allows a better understanding of the differences in pathogenicity and treatment response. A 57-year-old man with a history of B-cell lymphoma in remission was transferred from the hematology department due to fever of unknown origin, night sweats and asthenia. The empirical antibiotic therapy was initiated with no clinical response, and he developed a subacute pneumonia, severe anemia and hepatosplenomegaly. After positive blood, bronchoalveolar lavage and bone marrow cultures, a disseminated Mycobacterium avium-intracellulare complex infection was diagnosed, and the patient began treatment with clarithromycin, rifabutin and ethambutol. Two weeks later, a fourth antibiotic was added, amikacin at first and then linezolid, with slow but gradual improvement. Due to amikacin-related severe kidney injury and linezolid-related severe myelosuppression, the fourth antibiotic was changed to moxifloxacin, which the patient tolerated. After 6 months of therapy, the sensitivity to the regimen was confirmed and the species was identified as Mycobacterium chimaera (MC), using the molecular genetic test GenoType NTM-DR. The blood and tissue cultures were negative after 4 months of therapy, and treatment was continued for 12 months. Although the infection was being treated successfully, the patient's B-cell lymphoma relapsed after 12 months and the patient died. This is a case report of a confirmed severe and disseminated $\mathrm{MC}$ infection in an immunocompromised patient using a molecular genetic test, successfully treated using a four-drug regimen.
\end{abstract}

Keywords: Nontuberculous mycobacterium; Mycobacterium chime$\mathrm{ra}$; GenoType NTM-DR

\section{Introduction}

Mycobacterium chimaera (MC), a member of the Mycobac-

Manuscript submitted January 14, 2020, accepted January 21, 2020

aServico de Doencas Infeciosas do Centro Hospitalar Universitario de Coimbra, Coimbra, Portugal

bServico de Medicina Interna do Centro Hospitalar Universitario de Coimbra, Coimbra, Portugal

${ }^{\mathrm{c} C o r r e s p o n d i n g ~ A u t h o r: ~ R u b e n ~ d e ~ M e l o ~ C a r v a l h o, ~ S e r v i c o ~ d e ~ D o e n c a s ~ I n f e-~}$ ciosas, do Centro Hospitalar Universitario de Coimbra, 3004-561 Coimbra, Portugal. Email: ruben.melo.carvalho@gmail.com

doi: https://doi.org/10.14740/jmc3420 terium avium-intracellulare complex (MAC), is ubiquitous in the environment. It has been increasingly recognized as an emerging opportunistic threat specifically after open cardiac surgery and in immunocompromised patients [1].

The diagnosis is challenging due to nonspecific symptoms and an indolent course of the disease. It may present as pneumonia, endocarditis, implant-associated infection, hepatitis and chorioretinitis, as well as disseminated infection [2].

The pathogen is classified as MAC when cultured from blood and tissue samples. The species identification as MC is only possible using molecular genetic tests like the GenoType NTM-DR, a tool that also identifies genes responsible for some of the macrolides and aminoglycosides resistance mechanisms [3].

The current recommendations advocate that $\mathrm{MC}$ pulmonary disease should be treated with a three- to four-drug regimen, but recent evidence suggests disseminated infection may require four to five antibiotics depending on the severity of disease and patient risk factors, like kidney failure [4]. The regimen should include a macrolide, rifamycin, ethambutol, and a fourth agent such as moxifloxacin. The addition of a parenteral drug such as amikacin when starting treatment is also recommended [5].

We describe a case of a confirmed disseminated MC infection in an immunocompromised patient, with documented treatment efficacy using a four-drug regimen.

\section{Case Report}

A 57-year-old man presented with a 2-week history of lowgrade fever of unknown origin, night sweats, asthenia and severe weight loss. He was being followed in the hematology department due to a diffuse large B-cell lymphoma previously in remission for 9 years, which had relapsed 1 year before. He had been treated with rituximab, cyclophosphamide, doxorubicin, vincristine and prednisone (R-CHOP), and remission was confirmed again. He was expected to undergo autologous hematopoietic stem cell transplantation, which was postponed due to the febrile illness.

Empirical antibiotic therapy was started with a slow clinical response, and without microbiological identification at the beginning. One month later, the patient developed subacute pneumonia and severe anemia, as well as a new-onset hepatosplenomegaly ( $20 \mathrm{~cm}$ and $27 \mathrm{~cm}$, respectively).

Because a relapse of the lymphoma was suspected, the patient underwent liver and bone marrow biopsies, which showed no evidence of a hematological neoplasia. The liver biopsy 
identified a granulomatous hepatitis. After positive blood and bone marrow cultures, as well as a positive bronchoalveolar lavage culture, a disseminated MAC infection was diagnosed and the patient started treatment with clarithromycin, rifabutin and ethambutol (CRE).

After 2 weeks of therapy and due to clinical worsening, a fourth antibiotic was added, first amikacin for 2 weeks and subsequently linezolid for 3 weeks. Both had to be discontinued due to severe kidney injury and severe myelosuppression, respectively.

The antibiotic regimen (AR) was changed to CRE plus moxifloxacin, which was well tolerated, and the patient started to improve. The antimicrobial susceptibility testing (AST) was then available and revealed sensitivity to the CRE regimen, with resistance described only for streptomycin (amikacin and moxifloxacin resistance were not tested). The blood and tissue cultures were negative for MC after 4 months of therapy.

After 6 months of therapy, the identification of MC was possible using the newly available GenoType NTM-DR, a molecular genetic test that also detected no mutations at the rrs and $r r l$ genes (the former being responsible for some of the amikacin resistance and the latter for a potential macrolide resistance mechanism).

Although the infection was being treated, the autologous hematopoietic stem cell transplantation that should have followed R-CHOP was postponed due do the MC-related symptoms. Without it, the chances of a diffuse large B-cell lymphoma relapse were higher, and it happened after 12 months of the four-drug AR. A new chemotherapy regimen was being planned, but the patient died because of the lymphoma.

\section{Discussion}

Unlike many previous MC infection reports, our patient did not have a history of cardiac surgery or related procedures. However, MC has been increasingly recognized as the cause of opportunistic infections in immunocompromised patients. Our patient was immunocompromised due to a hematological neoplasia and the subsequent R-CHOP treatment. Following remission of the diffuse large B-cell lymphoma, he developed a $\mathrm{MC}$ disseminated infection.

As described in the literature, a four-drug AR was required, starting with CRE plus amikacin, later changed to CRE plus moxifloxacin due to amikacin-related kidney injury. The patient had a slow but gradual clinical improvement and the cultures were MC-negative after 4 months, confirming the efficacy of the four-drug AR.

The MC identification was possible using a molecular genetic test, the GenoType NTM-DR, which also excluded the existence of mutations for macrolide resistance in the $r r l$ gene, complementing the AST results.

The usefulness of this case is the description of a rare cause of disseminated infection in an immunocompromised patient, with good response to the recommended four-drug treatment and species identification using a molecular genetic test. In immunocompromised patients with a fever of unknown origin, this should be one of the etiologies to be taken into account.

\section{Acknowledgments}

None to declare.

\section{Financial Disclosure}

None to declare.

\section{Conflict of Interest}

None to declare.

\section{Informed Consent}

Informed consent was obtained from the patient for publication of this case report.

\section{Author Contributions}

Ruben de Melo Carvalho and Ana Luisa Nunes contributed to the writing of this manuscript; and all the authors were involved in the patient's treatment and the review of this report.

\section{Data Availability}

The data supporting the findings of this study are available from the corresponding author upon reasonable request.

\section{References}

1. Kasperbauer SH, Daley CL. Mycobacterium chimaera infections related to the heater-cooler unit outbreak: a guide to diagnosis and management. Clin Infect Dis. 2019;68(7):1244-1250.

2. Riccardi N, Monticelli J, Antonello RM, Luzzati R, Gabrielli M, Ferrarese M, Codecasa L, et al. Mycobacterium chimaera infections: An update. J Infect Chemother. 2019.

3. Huh HJ, Kim SY, Shim HJ, Kim DH, Yoo IY, Kang OK, $\mathrm{Ki} \mathrm{CS}$, et al. GenoType NTM-DR performance evaluation for identification of mycobacterium avium complex and mycobacterium abscessus and determination of clarithromycin and amikacin resistance. J Clin Microbiol. 2019;57(8).

4. Kasperbauer SH, Daley CL. Diagnosis and treatment of infections due to Mycobacterium avium complex. Semin Respir Crit Care Med. 2008;29(5):569-576.

5. Haworth CS, Banks J, Capstick T, Fisher AJ, Gorsuch T, Laurenson IF, Leitch A, et al. British Thoracic Society Guideline for the management of non-tuberculous mycobacterial pulmonary disease (NTM-PD). BMJ Open Respir Res. 2017;4(1):e000242. 